\title{
PRODUÇÁO DE HIDROGÊNIO A PARTIR DA REFORMA A VAPOR DO GLICEROL UTILIZANDO CATALISADORES DE NiCu OBTIDOS A PARTIR DE PRECURSORES TIPO-HIDROTALCITA
}

\author{
Robinson L. Manfro ${ }^{1}$ \\ Nielson F.P. Ribeiro ${ }^{1}$ \\ Mariana M.V.M. Souza ${ }^{I^{*}}$
}

\begin{abstract}
Resumo: O processo de produção do biodiesel gera aproximadamente $10 \%$ de glicerol como subproduto e esse processo tem aumentado progressivamente, o que gera excedente de glicerol no mercado. Estudos mostram a possibilidade de produção de hidrogênio através da reação de reforma a vapor do glicerol em temperaturas moderadas (em torno de $500^{\circ} \mathrm{C}$ ). Neste trabalho a reação de reforma a vapor do glicerol foi estudada utilizando três catalisadores derivados de compostos tipo-hidrotalcita com $20 \%$ em peso de $\mathrm{NiO}$ e 0,5 e $10 \%$ de $\mathrm{CuO}$. Os catalisadores foram caracterizados quanto à composiçáo química, estrutura cristalina, análise textural e redutibilidade. Os testes catalíticos foram realizados em reator de quartzo a pressão atmosférica utilizando uma velocidade espacial (GHSV) de $50.000 \mathrm{~h}^{-1}$ e soluçáo de glicerol a 10 vol\%. A conversão do glicerol foi praticamente de $100 \%$ a $500{ }^{\circ} \mathrm{C}$ e a composição gasosa, em termos de fração molar, ficou ao redor dos $65 \%$ para o $\mathrm{H}_{2}, 35 \%$ para o $\mathrm{CO}_{2}$ e baixos teores de $\mathrm{CO}$ e $\mathrm{CH}_{4}$. Na fase líquida foram identificados subprodutos como propilenoglicol, acetaldeído, ácido acético e etanol.
\end{abstract}

Palavras-chave: Hidrogênio, glicerol, reforma a vapor, hidrotalcita.

Abstract: The production of biodiesel produces approximately $10 \%$ of glycerol as a byproduct, and this process is increasing which generates a surplus of glycerol on the market. Studies show the possibility of producing hydrogen by steam reforming reaction of glycerol at moderate temperatures (about $500^{\circ} \mathrm{C}$ ). In this work the reaction of steam reforming of glycerol was performed using three hydrotalcite-type catalysts with $20 \mathrm{wt} \% \mathrm{NiO}$ and 0,5 and $10 \% \mathrm{CuO}$. The catalysts were characterized by chemical composition, crystalline structure, textural analysis and reducibility. The catalytic tests were performed in quartz reactor at atmospheric pressure using gas hourly space velocity (GHSV) of 50,000 $\mathrm{h}^{-1}$ and glycerol solution of 10 vol. \%. The conversion of glycerol was practically $100 \%$ at $500^{\circ} \mathrm{C}$ and the gas composition in terms of mole fraction was around $65 \%$ for $\mathrm{H}_{2}, 35 \% \mathrm{CO}_{2}$ and low levels of $\mathrm{CO}$ and $\mathrm{CH}_{4}$. In the liquid phase products were identified as propyleneglycol, acetaldehyde, acetic acid and ethanol.

Keywords: Hydrogen, glycerol, steam reforming, hydrotalcite.

1 Escola de Química-UFRJ, Centro de Tecnologia, Bloco E, sala 206, CEP 21941-909, Rio de Janeiro/RJ- Brasil.

*mmattos@eq.ufrj.br 


\section{INTRODUÇÃO}

A procura por fontes alternativas de energia encontra-se em crescente aumento no mundo motivado pelas previsóes que apontam para uma diminuiçáo progressiva da produçáo dos combustíveis fósseis. Além da escassez, outro problema associado ao uso de combustíveis fósseis são os aumentos contínuos das emissóes de poluentes, principalmente os relacionados com o aquecimento global. Estes gases poluentes afetam a saúde humana e também causam desequilíbrios na fauna e na flora, como a chuva ácida. Portanto, há uma grande necessidade de combustíveis alternativos que não afetem o meio ambiente (Shahid et al., 2008).

Para que o hidrogênio se torne uma fonte de energia realmente sustentável, deve-se promover a sua produção a partir de recursos renováveis, no entanto, mais de $95 \%$ do hidrogênio produzido atualmente provem de fontes não renováveis, baseados em combustíveis fósseis.

A produção de biodiesel a partir da transesterificação de óleos vegetais gera glicerol como subproduto. A produçáo de uma tonelada de biodiesel resulta em cerca de $110 \mathrm{~kg}$ de glicerol bruto ou $100 \mathrm{~kg}$ de glicerol puro (Adhikari et al., 2008). Segundo dados da ANP (Agência Nacional do Petróleo, Gás Natural e Biocombustíveis) em 2009 a produção brasileira de biodiesel (B100) foi de $1.608 .050 \mathrm{~m}^{3}$ gerando $171.829 \mathrm{~m}^{3}$ de glicerina (ANP, 2010), provocando um excedente de glicerina no mercado brasileiro. É esperado que a produção brasileira de biodiesel (B100) cresça ainda mais devido principalmente às obrigatoriedades do uso de biodiesel no óleo diesel, pois a partir de 01/01/2010, o biodiesel passou a ser adicionado ao óleo diesel na proporção de $5 \%$ em volume, conforme Resolução CNPE no 6 de 16/09/2009.

Cortright et al. (2002) e Davda et al. (2005) obtiveram hidrogênio a partir de compostos oxigenados derivados de biomassa incluindo o glicerol, através do processo de reforma em fase líquida (APR - Aqueous phase reforming). Embora o catalisador tenha apresentado estabilidade por um longo período, a alta pressão e baixas taxas de reaçáo tem impedido seu uso como um processo comercialmente viável. Por outro lado, o processo de reforma a vapor pode ser realizado a pressão atmosférica, com alta eficiência energética e com maior rentabilidade (Adhikari et al., 2007). A reação de reforma a vapor é altamente endotérmica e, idealmente, deve ser realizada a altas temperaturas, baixas pressóes, e alta razão vapor/glicerol para obter altas conversóes (Sehested, 2006).

A reforma a vapor do glicerol gera $\mathrm{H}_{2} \mathrm{e} \mathrm{CO}_{2}$ como principais produtos, com baixas concentraçóes de $\mathrm{CH}_{4}$ e $\mathrm{CO}$, porém ácidos orgânicos e alcanos também podem ser formados (Cortright et al., 2002). A reação global pode ser descrita de acordo com a Equaçáo 1:

$$
\mathrm{C}_{3} \mathrm{H}_{8} \mathrm{O}_{3}+3 \mathrm{H}_{2} \mathrm{O} \rightarrow 3 \mathrm{CO}_{2}+7 \mathrm{H}_{2} \text { (Eq.1) }
$$

As hidrotalcitas são conhecidas como minerais da família das argilas aniônicas e ainda como hidróxidos duplos lamelares (HDL). O interesse na síntese destes materiais decorre do fato de que a decomposição térmica dos compostos tipo-hidrotalcita leva a formação de óxidos mistos com alta dispersão metálica, alta área específica, estabilidade térmica e boa homogeneidade.

Catalisadores baseados em metais nobres possuem uma menor sensibilidade à deposição de carbono e maior atividade nas reaçóes de reforma. No entanto, considerando seu alto custo e sua limitada disponibilidade, é mais econômico do ponto de vista comercial desenvolver catalisadores baseados em metais não nobres, como o níquel, com bom desempenho e alta resistência à deposição de carbono.

Os catalisadores do tipo-hidrotalcita preparados neste trabalho apresentaram substituições dos cátions de magnésio por cátions de níquel e cobre, além dos cátions de alumínio. Com isso pretende-se desenvolver um catalisador com alta atividade na reação de reforma a vapor do glicerol e que produza uma fase gasosa com alta concentração de hidrogênio e com baixa concentração de monóxido de carbono.

\section{EXPERIMENTAL}

\subsection{PREPARAÇÃO DOS CATALISADORES}

Foram preparados três catalisadores tipo-hidrotalcita com $20 \%$ em peso de $\mathrm{NiO}$ e com diferentes teores de $\mathrm{CuO}$. Os catalisadores foram chamados de NiHT, Ni5CuHT e Ni10CuHT, contendo 0, 5 e $10 \%$ de $\mathrm{CuO}$, respectivamente, após a calcinação. O teor do níquel foi baseado no trabalho de Cruz (2006), que estudou a atividade dos catali- 
sadores a base de níquel na reforma do etanol em fase líquida, verificando que o melhor desempenho foi obtido para o teor de $20 \%$ de $\mathrm{NiO}$.

A metodologia de preparação utilizada foi baseada em Corma et al. (1994). Foram preparados $100 \mathrm{~mL}$ de uma solução $\mathrm{A}$, contendo os nitratos metálicos e $100 \mathrm{~mL}$ de uma solução $\mathrm{B}$ contendo quantidades apropriadas de $\mathrm{Na}_{2} \mathrm{CO}_{3} \mathrm{e}$ $\mathrm{NaOH}$. A solução A foi adicionada por gotejamento à solução $\mathrm{B}$, contida num reator de teflon, a uma taxa de $1 \mathrm{mLmin}^{-1}$, sob agitação vigorosa a temperatura ambiente. Em seguida, o gel formado permaneceu por $18 \mathrm{~h}$ a $60^{\circ} \mathrm{C}$. Terminado o tempo de envelhecimento, as amostras foram filtradas e lavadas a vácuo utilizando água destilada e deionizada na temperatura de $90^{\circ} \mathrm{C}$ até atingir o $\mathrm{pH}$ 7. Os compostos tipo-hidrotalcita foram então secos a $110^{\circ} \mathrm{C}$ durante uma noite. Por fim, os catalisadores foram calcinados a $500^{\circ} \mathrm{C}$ por 3 h, empregando-se uma taxa de aquecimento de $10^{\circ} \mathrm{Cmin}^{-1}$, sob fluxo de ar, numa vazáo de 60 $\mathrm{mLmin}^{-1}$.

\subsection{CARACTERIZAÇÃO DOS CATALISADORES}

Para determinação da composição química dos catalisadores foi utilizada a técnica de fluorescência de raios X (FRX) em um espectrômetro da marca Rigaku modelo RIX 3100, dotado de tubo gerador de raios $\mathrm{X}$ de ródio.

Medidas de difração de raios X (DRX) foram realizadas em um difratômetro da marca $\mathrm{Ri}$ gaku modelo Miniflex com radiação de $\mathrm{CuK} \alpha$ (30 kV e $15 \mathrm{~mA})$. O intervalo analisado foi de $5^{\circ} \leq 2 \theta \leq 90^{\circ}$ com passo de $0,05^{\circ}$, utilizando um tempo de contagem de 1 segundo por passo.

As propriedades texturais dos catalisadores foram determinadas por fisissorção de $\mathrm{N}_{2}$. As análises foram realizadas num equipamento ASAP modelo 2000 da Micromeritics. A área específica foi obtida utilizando o método de BET (Brunauer, Emmet e Teller) e o volume específico e o diâmetro de poros foram obtidos pelo método BJH a partir da isoterma de adsorção. As amostras foram primeiramente submetidas a um tratamento térmico de secagem a $200^{\circ} \mathrm{C}$ sob vácuo de $5 \times 10^{-3}$ torr, por um período de $24 \mathrm{~h}$.

A análise de redução à temperatura programada (TPR) foi realizada em equipamento convencional equipado com detector de condutivida- de térmica (TCD), usando uma mistura de 1,52\% $\mathrm{H}_{2} / \mathrm{Ar}$, e argônio puro como referência, com fluxo de $30 \mathrm{mLmin}^{-1}$. As amostras eram primeiramente submetidas à secagem com argônio por $30 \mathrm{~min}$ a $150{ }^{\circ} \mathrm{C}$ para remover a umidade. As amostras eram entáo resfriadas até a temperatura ambiente e em seguida iniciava-se o aquecimento até 1000 ${ }^{\circ} \mathrm{C}$, na taxa de $10{ }^{\circ} \mathrm{Cmin}^{-1}$, sob o fluxo da mistura redutora na vazáo de $30 \mathrm{mLmin}^{-1}$.

\subsection{TESTES CATALÍTICOS}

As reaçóes de reforma a vapor do glicerol foram realizadas a pressão atmosférica em um reator de quartzo de leito fixo usando $300 \mathrm{mg}$ de catalisador diluídos em $150 \mathrm{mg}$ material inerte (SiC) com tamanho de partícula similar. Antes de cada teste, os catalisadores foram reduzidos in-situ, empregando-se uma mistura redutora de $20 \% \mathrm{H}_{2} /$ $\mathrm{N}_{2}$ com fluxo de $75 \mathrm{mLmin}^{-1}$, aquecendo-se até $1000^{\circ} \mathrm{C}$ a uma taxa de $10^{\circ} \mathrm{Cmin}^{-1}$ e permanecendo por $30 \mathrm{~min}$. A soluçáo de 10 vol.\% de glicerol (PA - Vetec) em água foi alimentada a unidade reacional através de uma bomba Eldex modelo 1SM e vaporizada em fluxo de $\mathrm{He}$ em um reator de aço aquecido a $225^{\circ} \mathrm{C}$.

Os experimentos foram realizados a $500^{\circ} \mathrm{C}$ e com velocidade espacial horária (GHSV- Gas Hourly Space Velocity) de $50.000 \mathrm{~h}^{-1}$. O fluxo correspondente para $50.000 \mathrm{~h}^{-1}$ é de $0,121 \mathrm{~mL}$ $\min ^{-1}$ e a composição da fase gasosa é $20 \%$ vol. de $\mathrm{He}, 72$ \%vol. de água e 8 \%vol. de glicerol.

Antes das análises, os vapores gasosos da reação foram esfriados a $10{ }^{\circ} \mathrm{C}$ e com isso ocorria à separação das fases líquida e gasosa. A fase gasosa foi analisada on-line por cromatografia gasosa utilizando um cromatógrafo da Shimatzu, modelo GC - 2014, equipado com duas colunas (Rt-QPLOT e Carboxen 1010) e com detectores de condutividade térmica (TCD) e ionização de chama (FID). A fase líquida foi analisada em cromatografia líquida de alta performance (HPLC High-performance liquid chromatography), utilizando uma coluna da Bio-Rad modelo Aminex HPX-87H. 


\section{RESULTADOS E DISCUSSÁO}

\subsection{COMPOSIÇÃO QUÍMICA}

A composição química dos compostos tipo-hidrotalcita após calcinação é apresentada na Tabela 1. Nesta tabela observamos que a porcentagem de $\mathrm{NiO}$ e $\mathrm{CuO}$ está bastante próxima aos valores teóricos. No entanto, o catalisador NiHT foi o que apresentou uma maior diferença entre o valor nominal e real, tendo $16,7 \%$ de $\mathrm{NiO}$, ao invés dos $20 \%$. Essa perda pode estar associada ao processo de síntese, no qual o nitrato de níquel pode não ter sido completamente precipitado e/ou por perdas no preparo e manuseio das soluções.

Tabela 1. Resultados obtidos pela análise de FRX para os catalisadores calcinados.

\begin{tabular}{|c|c|c|c|c|}
\hline \multirow{2}{*}{ Amostra } & \multicolumn{4}{|c|}{ Teores mássicos dos óxidos (\%) } \\
\cline { 2 - 5 } & $\mathrm{NiO}$ & $\mathrm{CuO}$ & $\mathrm{Al} 2 \mathrm{O} 3$ & $\mathrm{MgO}$ \\
\hline $\mathrm{NiHT}$ & 16,7 & - & 25,8 & 57,5 \\
\hline $\mathrm{Ni}$ CuHT & 19,1 & 5,3 & 27,5 & 48,0 \\
\hline Ni10CuHT & 19,2 & 11,3 & 24,6 & 44,8 \\
\hline
\end{tabular}

\subsection{DIFRAÇÃO DE RAIOS X}

Os difratogramas de raios $\mathrm{X}$ (DRX) dos precursores tipo-hidrotalcita são apresentados na Figura 1. Os principais picos encontrados estáo localizados nos ângulos $2 \theta$ de $11,5^{\circ}, 22,9^{\circ}, 34,6^{\circ}, 60,5^{\circ}$ e $61,9^{\circ}$, comprovando que as amostras possuem como fase precursora compostos tipo-hidrotalcita (JCPDS 41-1428). A ausência de outras fases sugere que tanto o $\mathrm{Ni}^{+2}$ quanto o $\mathrm{Cu}^{+2}$ substituíram isomorficamente os cátions $\mathrm{Mg}^{+2}$ nas camadas tipo-brucita, o que está relacionado com a semelhança entre os raios iônicos do $\mathrm{Ni}^{2+}(0,72 \AA)$, $\mathrm{Cu}^{2+}(0,73 \AA)$ e $\mathrm{Mg}^{2+}(0,65 \AA)$.

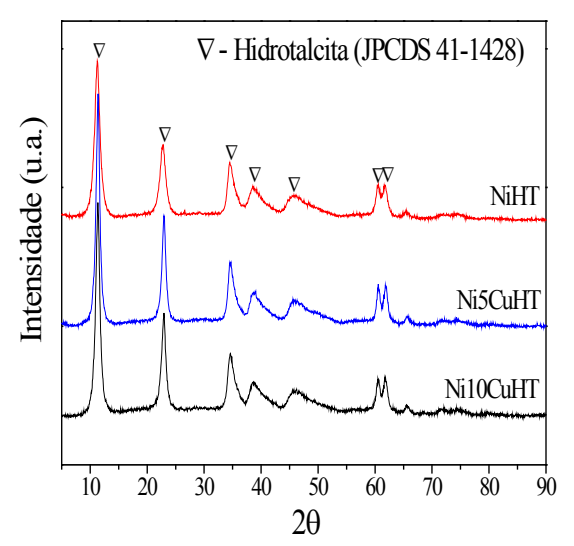

Figura 1. Difratogramas das amostras sintetizadas.

Após a calcinação, os difratogramas (Figura 2) mostram que a amostra $\mathrm{NiHT}$ apresentou a formação de óxidos mistos gerados pela destruição da estrutura lamelar, sendo caracterizada pelo desaparecimento dos picos característicos da hidrotalcita, e a segregação de uma fase $\mathrm{MgO}$ periclásico $\left(42,9^{\circ}, 62,3^{\circ}\right.$ e 78,6 $6^{\circ}$ JCPDS 45-0946). Este resultado indica que ambos os óxidos de níquel e alumínio estão bem dispersos na matriz de $\mathrm{MgO}$, sem segregação de uma fase de espinélio. Já as amostras Ni5CuHT e Ni10CuHT, além da fase $\mathrm{MgO}$ periclásico, apresentaram a formação de uma solução sólida composta por $\left(\mathrm{Ni}_{(1-\mathrm{x})}-\mathrm{Cu}_{\mathrm{x}}\right)$ O (JCPDS - 25-1049), que possui picos nos ângulos $2 \theta$ de $37,1^{\circ}, 43,2^{\circ}$ e $62,7^{\circ}$.

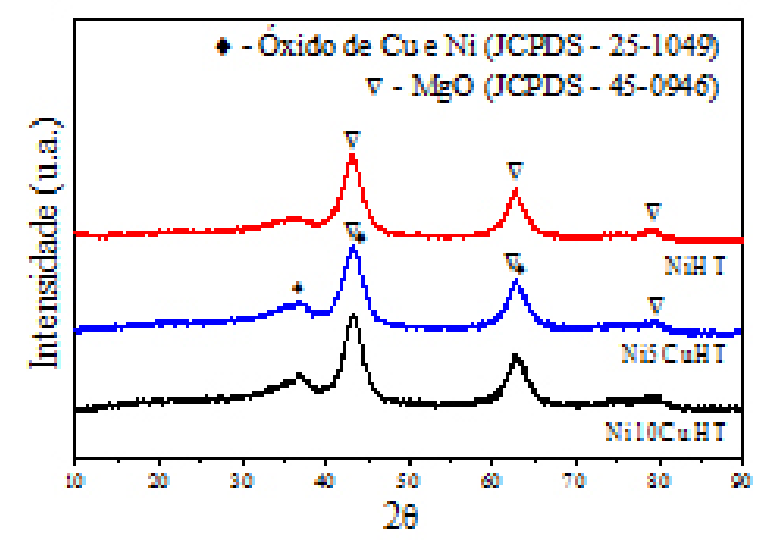

Figura 1. Difratogramas das amostras calcinadas a $500^{\circ} \mathrm{C}$.

\subsection{ANÁLISE TEXTURAL}

A Tabela 2 apresenta os valores de área específica e volume de poros das amostras preparadas. A área específica ficou na faixa de 286 a $203 \mathrm{~m}^{2} \mathrm{~g}^{-1}$ para os catalisadores calcinados, e na faixa de 42 a $88 \mathrm{~m}^{2} \mathrm{~g}$ ${ }^{1}$ para os precursores náo calcinados. A porosidade 
dos catalisadores aumenta após a calcinação devido a dois diferentes fatores. Um deles é devido ao surgimento de porosidade intrapartícula que ocorre após a calcinação, o qual está associado à remoçáo de água e dos íons carbonatos, sob a forma de $\mathrm{CO}_{2}$, do espaço interlamelar, provocando a destruiçáo da estrutura lamelar, como pode ser observado nos difratogramas de raios X (López et al., 1997). Outro fator é devido a um empilhamento irregular de partículas criando poros interpartículas. $\mathrm{O}$ tamanho dos poros interpartículas depende principalmente do tamanho do cristal e a porosidade interpartícula contribui bastante para o volume total de poro (Valente et al., 2010).

A adição de cobre provoca uma reduçáo da área específica e do volume de poro dos catalisadores calcinados. Estas modificaçóes nas propriedades texturais dos catalisadores podem estar associadas ao fato do óxido de cobre formar uma solução sólida com o óxido de níquel, após a calcinação, resultando na formação de aglomerados de menor área específica e volume de poro.

Tabela 2. Características texturais das amostras calcinadas e não calcinadas.

\begin{tabular}{|c|c|c|}
\hline Amostra & $\mathrm{S}_{\left.\mathrm{BET}_{1}\right)}\left(\mathrm{m}^{2} \mathrm{~g}^{-}\right.$ & $\mathrm{V}_{\text {poro }}\left(\mathrm{cm}^{3} \mathrm{~g}^{-1}\right)$ \\
\hline NiHT* & 286 & 0,70 \\
\hline NiHT & 88 & 0,40 \\
\hline Ni5CuHT* & 211 & 0,48 \\
\hline Ni5CuHT & 42 & 0,16 \\
\hline Ni10CuHT* & 203 & 0,41 \\
\hline Ni10CuHT & 60 & 0,15 \\
\hline
\end{tabular}

* Amostras Calcinadas

\subsection{REDUÇÃO A TEMPERATURA PROGRAMADA (TPR)}

A Figura 3 apresenta os perfis da redução a temperatura programada (TPR) das amostras calcinadas. A amostra NiHT apresenta um único pico de redução em $895^{\circ} \mathrm{C}$, que corresponde a redução de espécies de níquel (níquel em solução sólida com o magnésio e/ou aluminato de níquel) a níquel metálico. Já as amostras Ni5CuHT e Ni10CuHT apresentam dois picos de reduçáo distintos, pois o óxido de cobre possui uma temperatura de redução na faixa dos 200 a $400^{\circ} \mathrm{C}$ (Dussault et al.,
2005). Desta forma é possível calcular o grau de redução para cada óxido, Tabela 3.

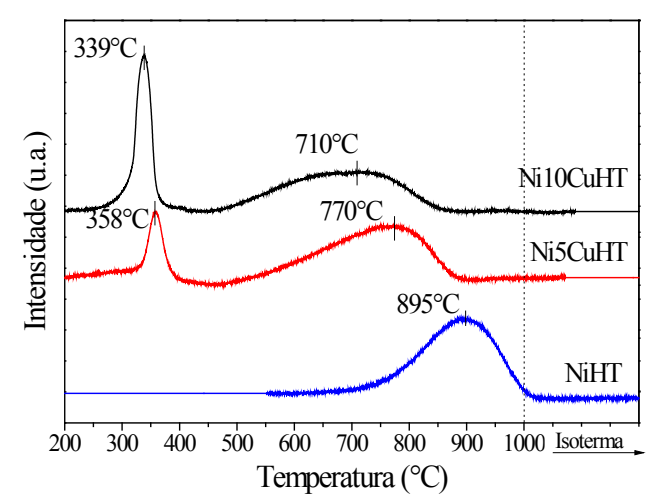

Figura 3. Perfis de TPR das amostras calcinadas.

Tabela 3. Grau de redução (\%) dos óxidos de cobre e de espécies de níquel.

\begin{tabular}{|c|c|c|}
\hline Amostra & $\mathrm{CuO}$ & $\mathrm{NiO}$ \\
\hline $\mathrm{NiHT}$ & - & 72 \\
\hline Ni5CuHT & 79 & 107 \\
\hline Ni10CuHT & 94 & 100 \\
\hline
\end{tabular}

Pode-se observar na Figura 3 que o aumento do teor de cobre resulta em uma diminuição da temperatura máxima de redução do níquel e do cobre. Isto pode estar relacionado ao efeito de spillover do hidrogênio, o qual acelera a nucleação do níquel metálico, nestas condiçôes de redução, além disso, aumenta a redutibilidade (Ashok et al., 2008). Na Tabela 3 podemos verificar que a presença de cobre aumenta a redutibilidade do níquel.

As amostras Ni5CuHT e Ni10CuHT apresentam uma faixa de redução entre 250 a $430^{\circ} \mathrm{C}$ correspondendo a redução do óxido de cobre a cobre metálico, com picos máximos de redução em 358 a $339^{\circ} \mathrm{C}$, respectivamente. Estas amostras apresentam em uma faixa mais alta de temperatura, que se estende dos 500 a $950^{\circ} \mathrm{C}$, a redução das espécies de níquel, as quais apresentam pico máximo de redução em 770 e $710^{\circ} \mathrm{C}$, respectivamente.

A amostra NiHT apresenta um grau de redução pouco maior que $72 \%$. Este menor grau de redução pode ser associado à redução das espécies $\mathrm{NiAl}_{2} \mathrm{O}_{4}$ formadas em temperaturas superiores a $800^{\circ} \mathrm{C}$ durante a análise de TPR ou dos átomos de níquel localizados em uma solução sólida composta por $\mathrm{Mg}-\mathrm{Ni}-\mathrm{O}$, o qual proporciona uma grande estabilidade aos íons de níquel resultando 
em altas temperaturas de redução (Schulze et al., 2001).

\subsection{TESTES CATALÍTICOS}

De acordo com estudos termodinâmicos o rendimento máximo em $\mathrm{H}_{2}$ é obtido ao redor de $650^{\circ} \mathrm{C}$ na reforma a vapor do glicerol (Adhikari et al., 2007; Velu et al., 1999). Tendo em vista o consumo energético e o bom desempenho obtido a $500{ }^{\circ} \mathrm{C}$, no qual a conversão foi de praticamente $100 \%$, a fraçáo molar do $\mathrm{H}_{2}$ ficou ao redor de $63 \%$ e do $\mathrm{CO}_{2}$ em $34 \%$, apresentando fraçóes molares de $\mathrm{CH}_{4}$ e $\mathrm{CO}$ menores que $1 \%$, optou-se em trabalhar nesta temperatura. Apesar da limitação termodinâmica em se trabalhar com conversão próxima a $100 \%$, diferentes seletividades a produtos gasosos e líquidos foram obtidas com os diferentes catalisadores, permitindo assim distingui-los quanto à formação dos diferentes produtos.

Selecionada a temperatura, realizaram-se testes catalíticos nas condiçôes já descritas, utilizando os catalisadores, NiHT, Ni5CuHT e Ni10CuHT. A Figura 4 apresenta as fraçóes molares percentuais de $\mathrm{H}_{2}, \mathrm{CO}_{2}, \mathrm{CO}$ e $\mathrm{CH}_{4}$ obtidas nos testes catalíticos. Estes são os únicos gases resultantes da reaçáo com o catalisador $\mathrm{NiHT}$, no entanto, a partir da quinta e terceira hora de reação com os catalisadores Ni5CuHT e Ni10CuHT, respectivamente, ocorreu o surgimento de outros elementos gasosos, os quais não foram identificados, no entanto, segundo a literatura (Adhikari et al., 2007; Iriondo et al., 2010) é possível que seja etano, propano, etileno e propileno.

Analisando a Figura 4, pode-se observar que para os três catalisadores a fraçáo molar do $\mathrm{H}_{2}$ ficou acima de $60 \%$. O catalisador NiHT apresentou um desempenho levemente superior frente aos demais, chegando a fraçóes molares ao redor dos $65 \%$ a partir da quinta hora de reação. A fração molar do $\mathrm{CO}_{2}$ para os três catalisadores ficou na faixa dos 33 a $38 \%$, no entanto, o catalisador Ni10CuHT teve a partir da quarta hora de reação uma redução para $30 \%$, no mesmo instante apresentou um aumento na fração molar do $\mathrm{CO}$ de 1 para $7 \%$ e uma reduçáo do $\mathrm{CH}_{4}$ de 3 para $1 \%$. $\mathrm{O}$ aumento do $\mathrm{CO}$ pode estar associado a uma redução da atividade na reação de shift e a redução do $\mathrm{CH}_{4}$ a uma redução na atividade da reaçáo de metanação e isto pode ser uma explicação para a relativa estabilidade do $\mathrm{H}_{2}$ durante todo o período da reação. A fração molar do $\mathrm{CO}$ e do $\mathrm{CH}_{4}$ para os catalisadores $\mathrm{NiHT}$ e $\mathrm{Ni}$ C CuHT não apresentou variaçóes significativas, ficando ao redor de 1 e $0,5 \%$ para o catalisador $\mathrm{NiHT}$ e 1,5 e $2 \%$ para o catalisador $\mathrm{Ni} 5 \mathrm{CuHT}$, respectivamente.

Figura 4. Fraçôes molares percentuais de $\mathrm{H}_{2}, \mathrm{CO}_{2}$, $\mathrm{CO}$ e $\mathrm{CH}_{4}$ obtidos na reforma a vapor do glicerol com solução a 10 vol. $\%$, temperatura de $500^{\circ} \mathrm{C}$, fluxo de $0,121 \mathrm{mLmin}^{-1}$
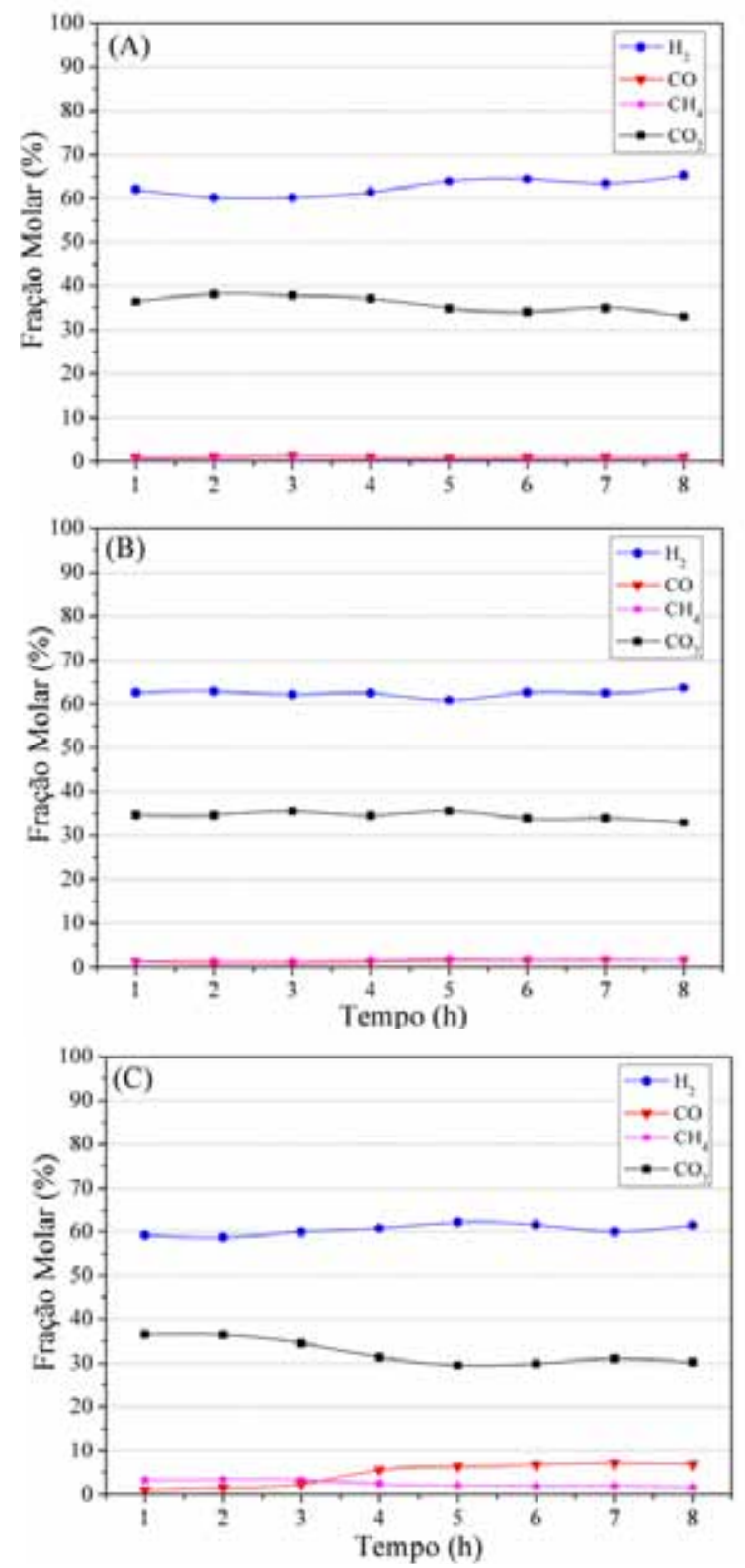

(50.000 h-1 GHSV) e $300 \mathrm{mg}$ de catalisador NiHT (a), Ni$5 \mathrm{CuHT}$ (b) e Ni10CuHT (c).

Os subprodutos formados na fase líquida (ácido acético, acetaldeído, propilenoglicol e etanol) foram identificados e quantificados, e são apresentados na Figura 5. A conversão do glicerol foi praticamente total para todos os catalisadores. Traços de outros subprodutos líquidos foram observados, porém não foram identificados. 
Figura 5. Conversão do glicerol e concentração de ácido acético, acetaldeído, propilenoglicol e etanol na fase liquida nos testes catalíticos realizados com catalisador NiHT (a), Ni5CuHT (b) e Ni10CuHT (c).
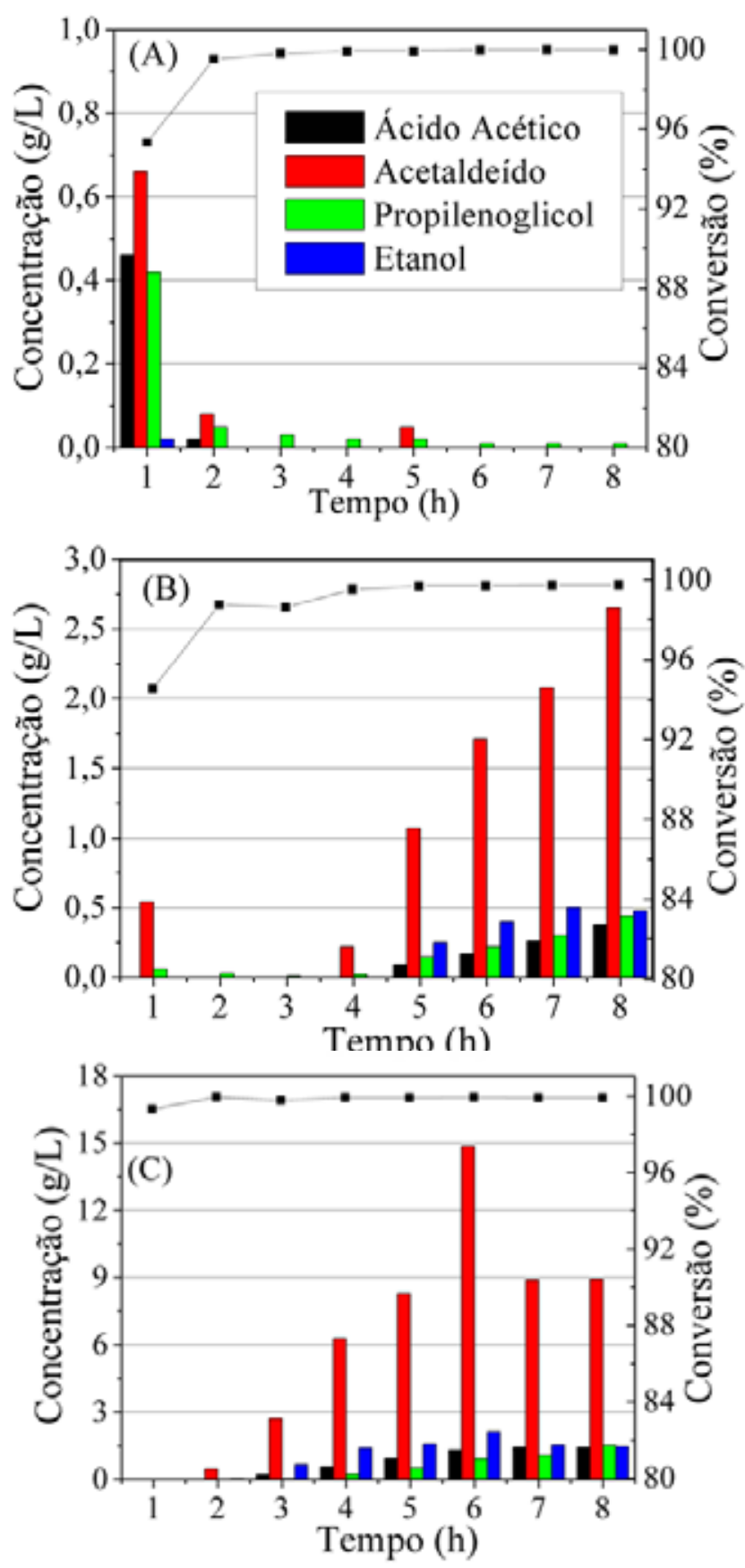

$\mathrm{Na}$ Figura 5 (a), referente ao teste realizado com o catalisador NiHT, verificamos a baixa formação de subprodutos líquidos, sendo encontrado concentraçóes pequenas na primeira hora de reação e nas horas seguintes apenas traços ou os subprodutos não foram formados.

Os subprodutos formados no teste com o catalisador Ni5CuHT, Figura 5 (b), apresentam uma tendência crescente a partir da quarta hora de reação, com destaque para o acetaldeído, o qual parte de aproximadamente 0,25 para mais de $2,5 \mathrm{gL}^{-1}$ no final da oitava hora de reaçáo. Os demais subprodutos apresentaram concentraçóes inferiores a $0,5 \mathrm{gL}^{-1}$.

A maior concentração de subprodutos líquidos foi obtida com o catalisador $\mathrm{Ni10CuHT}$, Figura 5 (c). Nas horas iniciais da reação praticamente náo houve a formaçáo de subprodutos, somente a partir da terceira hora de reação, com exceção do acetaldeído. A concentração de ácido acético, propilenoglicol e etanol ficaram na faixa de 0,5 a $2 \mathrm{gL}^{-1}$ a partir da quarta hora. $\mathrm{O}$ acetaldeído apresenta a maior de todas as concentraçóes encontradas chegando a aproximadamente $15 \mathrm{gL}^{-1}$ na sexta hora de reação, mas apresenta concentraçôes entre a terceira hora e a oitava hora de 3 a $9 \mathrm{gL}^{-1}$.

\section{CONCLUSÓES}

A composição química real dos catalisadores ficou bastante próxima da nominal, mostrando que o método de preparo é eficiente para a síntese dos catalisadores. Além disso, as análises de DRX mostraram que ocorreu de fato a formação das estruturas tipo-hidrotalcita com a incorporação do níquel e do cobre na estrutura.

Após a calcinação, todos os catalisadores apresentaram um aumento da área específica e do volume de poros, devido à destruição das estruturas lamelares dos compostos tipo-hidrotalcita. Observou-se que adição de cobre nos catalisadores ocasionava uma reduçáo nestas duas propriedades texturais, possivelmente devido a formação de soluçôes sólidas do cobre com outros metais.

A análise de TPR mostrou que o catalisador $\mathrm{NiHT}$ possui as maiores temperaturas de redução, devido a formação de aluminato de níquel e/ou soluçóes sólidas de $\mathrm{Mg}-\mathrm{Ni}-\mathrm{O}$. O aumento do teor de cobre nas amostras Ni5CuHT e Ni10CuHT aumenta a redutibilidade do níquel e do cobre e diminui a temperatura máxima de redução.

Todos os catalisadores apresentaram praticamente $100 \%$ de conversão do glicerol a partir da segunda hora de reação a $500{ }^{\circ} \mathrm{C}$. Durante as 8 $\mathrm{h}$ de duração dos testes catalíticos os catalisadores mostraram-se estáveis, não sofrendo desativação. A composiçáo gasosa é composta principalmente por $\mathrm{H}_{2}$ e $\mathrm{CO}_{2}$, apresentando baixas concentraçóes de $\mathrm{CH}_{4}$ e CO. O subproduto líquido principal 
formado é o acetaldeído. Foi observado que os catalisadores compostos por cobre apresentavam uma maior formação de subprodutos indesejados tanto na fase gasosa, os quais não foram identificados, como na fase líquida. Apesar do cobre apresentar alta atividade catalítica na reação de shift, observou-se uma influência negativa na reação de reforma a vapor do glicerol.

\section{AGRADECIMENTOS}

Os autores agradecem ao Núcleo de Catálise/COPPE/UFRJ pela análise de FRX e pelas caracterizaçóes texturais dos catalisadores. $\mathrm{E}$ ao $\mathrm{CNPq}$ pelo suporte financeiro.

\section{REFERÊNCIAS}

Adhikari, S., Fernando, S. D., Haryanto, A. Hydrogen production from glycerin by steam reforming over nickel catalysts. Renewable Energy, v.33, p.1097-1100, 2008.

Adhikari, S., Fernando, S. D., Haryanto, A. Production of hydrogen by steam reforming of glycerin over alumina-supported metal catalysts. $\mathrm{Ca}$ talysis Today, v.129, p.355-364, 2007.

Adhikari, S., Fernando, S., Gwaltney, S. R., Filip To, S. D., Bricka, R. M., Steele, P. H., Haryanto, A. A thermodynamic analysis of hydrogen production by steam reforming of glycerol. International Journal of Hydrogen Energy, v.32, p.28752880, 2007.

Ashok, J., Subrahmanyam, M., Venugopal, A. Hydrotalcite structure derived $\mathrm{Ni}-\mathrm{Cu}-\mathrm{Al}$ catalysts for the production of $\mathrm{H}_{2}$ by $\mathrm{CH}_{4}$ decomposition. International Journal of Hydrogen Energy, v.33, p.2704-2713, 2008.

Chiodo, V., Freni, S., Galvagno, A., Mondello, N., Frusteri, F. Catalytic features of $\mathrm{Rh}$ and $\mathrm{Ni}$ supported catalysts in the steam reforming of glycerol to produce hydrogen. Applied Catalysis A: General, v.381, p.1-7, 2010.

Corma, A., Fornes, V., Rey, F. Hydrotalcites as base catalysts - influence of the chemical-composition and synthesis conditions on the dehydroge- nation of isopropanol. Journal of Catalysis, v.148, p.205-212, 1994.

Cortright, R. D., Davda, R. R.,; Dumesic, J. A. Hydrogen from catalytic reforming of biomass-derived hydrocarbons in liquid water. Nature, v.418, p.964-967, 2002.

Cruz, I. O. Reforma do etanol em fase líquida utilizando catalisadores a base de hidrotalcitas. Dissertação de mestrado, Escola de Química - UFRJ, 2006.

Davda, R. R., Shabaker, J. W., Huber, G. W., Cortright, R. D., Dumesic, J. A. A review of catalytic issues and process conditions for renewable hydrogen and alkanes by aqueous-phase reforming of oxygenated hydrocarbons over supported metal catalysts. Applied Catalysis B: Environmental, v.56, p.171-186, 2005.

Dussault, L., Dupina, J. C., Dumitriub, E., Aurouxc, A., Guimona, C. Microcalorimetry, TPR and XPS studies of acid-base properties of $\mathrm{NiCu}$ $\mathrm{MgAl}$ mixed oxides using $\mathrm{LDH}$ s as precursors. Thermochimica Acta, v.434, p.93-99, 2005.

Iriondo, A., Barrio, V. L., Cambra, J. F., Arias, P. L., Guemez, M. B., Sanchez-Sanchez, M. C., Navarro, R. M., Fierro, J. L. G. Glycerol steam reforming over Ni catalysts supported on ceria and ceria-promoted alumina. International Journal of Hydrogen Energy, v.35, p.11622-11633, 2010.

López, T., Bosch, P., Asomoza, M., Gómez, R., Ramos, E. DTA-TGA and FTIR spectroscopies of sol-gel hydrotalcites: Aluminum source effect on physicochemical properties. Materials Letters, v.31, p.311-316, 1997.

Schulze, K., Makowski, W., Chyzy, R. D., Geismar, G. Nickel doped hydrotalcites as catalyst precursors for the partial oxidation of light paraffins. Applied Clay Science, v.18, p.59-69, 2001.

Sehested, J. Four challenges for nickel steam-reforming catalysts. Catalysis Today, v.111, p.103110, 2006. 
Shahid, E. M., Jamal, Y. A review of biodiesel as vehicular fuel. Renewable and Sustainable Energy Reviews, v.12, p.2484-2494, 2008.

Valente, J. S., Hernandez-Cortez, J., Cantu, M. S., Ferrat, G., López-Salinas, E. Calcined layered double hydroxides $\mathrm{Mg}-\mathrm{Me}-\mathrm{Al}$ (Me: $\mathrm{Cu}, \mathrm{Fe}$, $\mathrm{Ni}, \mathrm{Zn}$ ) as bifunctional catalysts. Catalysis Today, v.150, p.340-345, 2010.
Velu, S., Suzuki, K., Okazaki, M., Osaki, T., Tomura, S., Ohashi, F. Synthesis of new Sn-incorporated layered double hydroxides and their thermal evolution to mixed oxides. Chemistry of Materials, v.11, p.2163-2172, 1999. 